\title{
La práctica como investigación: nuevas metodologías para la academia latinoamericana
}

María José Contreras Lorenzini*

\begin{abstract}
RESUMEN: Muchas de las teorizaciones que en las últimas décadas han reconocido la relevancia sociocultural e histórica del cuerpo han privilegiado una mirada objetivante que considera el cuerpo como objeto de estudio. En esta presentación introduzco la metodología emergente denominada "práctica como investigación" que busca validar el cuerpo como un agente cognoscitivo por derecho propio promoviendo investigaciones académicas guiadas por la práctica donde el cuerpo con sus quehaceres y saberes es el principal agente movilizador. En el artículo discuto las implicancias epistemológicas, metodológicas y políticas de esta aproximación sosteniendo que esta perspectiva permite un giro epistémico que no se contenta con teorizar el cuerpo sino que busca ampliar el horizonte epistémico a través del cuerpo.
\end{abstract}

Palabras clave: cuerpo, investigación. práctica

\footnotetext{
* María José Contreras (Doctora en Semiótica, Universidad de Bolonia) es una artista de la performance y directora teatral Chilena. Es profesora asistente en la Escuela de Teatro de la Universidad Católica de Chile donde enseña en pregrado y postgrado. Su trabajo transita entre la investigación académica y la creación artística, estudiando y explorando desde las artes performativas la relación entre cuerpo, memoria y performance. Entre sus trabajos más recientes se encuentra la obra de teatro "Pajarito Nuevo la LLeva" basada en testimonios de niños durante la dictadura (próxima a publicarse por Bloomsbury, Londres), "\#quererNOver" una acción de arte masiva donde participaron más de 1.200 personas para recordar a los detenidos desaparecidos en Chile y "Habeas Corpus" la primera performance realizada en el Palacio de Tribunales de Chile que planteó una crítica sobre el rol de la justicia durante la dictadura en Chile. Sus artículos han sido publicados en Chile, Brasil, Reino Unido e Italia. Más información en www. mariajosecontreras.com
} 
Hasta la primera mitad del siglo XX el cuerpo era considerado una suerte de excedente epistémico al que no se le prestaba mucha atención. La herencia platónica marcó la teoría del conocimiento occidental que insiste en la distinción y jerarquización de la mente sobre el cuerpo, del saber (racional y verdadero) sobre el hacer (material y efímero). Los esclavos en la caverna de Platón, limitados a aquello que sus sentidos le mostraban, estaban condenados a no acceder a la verdad. Esta visión fundacional establece que los sentidos llevan a equívocos oponiéndose a la razón como camino exclusivo para acceder al conocimiento y la verdad. En el siglo XVII la llustración operó el distanciamiento definitivo respecto a la religión y el mito lo que reforzó el menoscabo de todos los conocimientos que no fueran racionales y científicos. Tal como planteó David Le Breton (2002) de la mano de Descartes la filosofía moderna terminó por cristalizar la hegemonía del pensamiento racional por sobre el conocimiento práctico. Este racionalismo y cientificismo han determinado aquello que se sanciona hegemónicamente como "conocimiento", fundando las premisas epistemológicas y metodológicas de la universidad en Occidente.

En las últimas décadas, sin embargo, disciplinas como la sociología, antropología y psicología, entre otras, se han abocado a estudiar las prácticas entendidas como articulaciones localmente determinadas de interacción entre cuerpos (humanos y no humanos). Campos de estudio como el feminismo, la teoría queer, los estudios críticos y culturales, así como los estudios de la performance, han avanzado en el estudio de las prácticas como formas de vinculación material entre los sujetos socioculturales. El estudio de las prácticas ha permitido indagar ese intersticio que permanecía como un "resto" entre las mentes individuales y los sistemas sociales, generando una nueva mirada sobre cómo se construye el conocimiento y se organiza la vida social. Schatzki, Knorr \& Von Savigny (2001), de hecho, han postulado una suerte de "giro de las prácticas" (practice turn) definido como una nueva ontología del social entendido como red de prácticas encarnadas que se configuran entre sujetos, artefactos y objetos ${ }^{1}$. Este giro de las prácticas se produjo en distintos campos del saber por lo que no constituye un movimiento homogéneo; sin embargo, las distintas perspectivas adhieren a la premisa general que establece que el conocimiento, el significado, la ciencia, el poder y el lenguaje ocurren en y por las prácticas.

Un nudo central, además, de los teóricos de las prácticas es la concepción de las prácticas como corporizadas [embodied], una colección de actividades humanas mediadas materialmente y 
organizadas en torno a un conocimiento práctico compartido. La calificación de "corporizadas" [embodied] se refiere, como muchos de los pensadores de finales del siglo veinte enfatizan (sobre todo feministas), a las formas como la actividad humana se entrelaza con las características del cuerpo humano. Aquellos teóricos de las prácticas que destacan la corporización [embodiment] creen típicamente que los cuerpos y las actividades se construyen en las prácticas. [...] Según estos teóricos las prácticas determinan y constituyen el contexto en el que las propiedades corporales cruciales para la vida social se forman, no solo en cuanto competencias y actividades, sino también como experiencias corporales, presentaciones de superficie, e incluso como estructuras física (SCHATZKI et. al., 2001, p. 11, trad. mía).

La atención por las prácticas ha permitido orientar muchos estudios hacia la comprensión de los modos de aparición y representación del cuerpo en la cultura. Sin duda, estas teorizaciones han contribuido a reposicionar el cuerpo para reconocer en sede teórica su valor en la construcción de la identidad, los imaginarios culturales y por supuesto también en la comunicación y vinculación de los sujetos socioculturales. El cuerpo ha devenido un objeto que articula múltiples miradas y perspectivas, funcionando incluso como una categoría de análisis transversal que permite la convergencia y diálogo entre disciplinas disímiles.

\section{El cuerpo como locus de conocimiento}

En este artículo postulo que el interés por el cuerpo y las prácticas no ha conllevado una validación del cuerpo como agente activo del conocimiento. La gran cantidad de teorizaciones que han reconocido la relevancia sociocultural e histórica del cuerpo lo consideran como un objeto de estudio y lo estudian por tanto desde una mirada objetivante. El cuerpo y sus saberes han entrado a la academia para ocupar un lugar análogo al del cadáver en los teatros anatómicos del siglo XV: al analizar el cuerpo la teoría contemporánea lo desprovee de su turgente carnalidad. Las universidades, en cuanto instituciones encargadas de distribuir y regular el conocimiento, escasamente validan los saberes del cuerpo como conocimientos de igual jerarquía que los denominados conocimientos científicos. En este artículo introduciré una perspectiva que se ha desarrollado sobretodo en la academia anglosajona vinculada a las artes que ha intentado superar la mirada objetivante sobre el cuerpo para promover metodologías de investigación guiadas por la práctica. Estas metodologías que instalan el cuerpo como principal agente cognoscitivo y experiencial han proliferado exponencialmente desde finales de 
los años setenta. Las facultades vinculadas a las artes (escuelas de teatro, de performance, de artes visuales, de música, entre otras) que tradicionalmente han conjugado los saberes prácticos propios de los oficios de los artistas con la reflexión teórica han sido el terreno más fértil para el desarrollo de estas metodologías. Las metodologías guiadas por la práctica proponen un giro epistémico importante que promueven una política del conocimiento nueva. Me parece que el campo de las artes puede hoy aportar estas reflexiones epistemológicas y políticas a otros campos disciplinarios. Espero con este escrito contribuir a forjar un puente que desde mi ámbito de competencia -las artes performativas- pueda aportar a la discusión sobre la investigación a condición de cuerpo en otros campos disciplinarios.

Las metodologías de investigación guiadas por la práctica desarrolladas en el ámbito de la investigación artística buscan no solo estudiar la corporalidad sino también la validación política de los conocimientos generados por y a través del cuerpo. Como mencioné anteriormente, estas apuestas metodológicas intentan resistir el imperialismo del discurso verbal en la academia para generar un auténtico giro epistémico que incluya los conocimientos no verbales. El cuerpo, en este contexto, deja de ser un objeto de estudio para devenir en un agente cognoscitivo capaz de generar conocimientos que exceden el lenguaje verbal y las codificaciones matemáticas que tanto ha privilegiado la ciencia moderna.

Como ya mencioné estas metodologías guiadas por la práctica se han desarrollado principalmente en la academia anglosajona, lo que ha implicado que su difusión se realice sobre todo en inglés. Me parece que estos modelos anglosajones pueden servir como referencia y contraste respecto al estado del arte en América Latina. A partir de la reflexión que planteo, discutiré la aplicabilidad y usabilidad en nuestros contextos locales de este tipo de metodologías, tanto aquellos vinculados con la investigación artística en las artes como en modo más general a la investigación guiada por la práctica.

Tal como revisa Sullivan (2006) términos como investigación basada en las artes (arts-based research, BARONE \& EISNER, 1997; EISNER, 2007), investigación informada por las artes (artsinformed research, COLE, NEILSON, KNOWLES \& LUCIANI, 2004) e investigación basada en la práctica (FRAYLING, 1997) han surgido de distintas filiaciones y contextos. La modalidad de investigación basada en las artes se asocia en general al ámbito de la educación y pretende revelar la importancia de las artes en los procesos de aprendizaje y enseñanza. En este 
documento me interesa el otro ámbito definido ampliamente como investigación basada en la práctica o investigación conducida por la práctica que define un tipo de investigación realizada en programas de postgrado que consideran el trabajo de taller como crucial en el desarrollo investigativo. Estos modelos de investigación surgen desde la necesidad a nivel de postgrado de incluir la práctica artística como modalidad para generar conocimientos "otros". En el Reino Unido, desde inicios de los noventa, se ha empezado a validar este tipo de metodologías, por ejemplo aceptando la exhibición de una obra creativa como parte del examen doctoral. Tal como explica Vera (2010) existen al menos cincuenta programas doctorales en Australia, Canadá, Sudáfrica, Estados Unidos y Reino Unido que contemplan la posibilidad de presentar una obra como resultado final. Estos programas que admiten resultados prácticos trabajan desde la conjunción de la teoría y la práctica lo que se manifiesta en todos los niveles de los proyectos doctorales: desde su diseño, pasando por sus (inéditas) metodologías y finalmente por la producción de resultados que pueden ser prácticos (en el caso de una obra de artes visuales como resultado doctoral) o mixtos (que considera la obra más un documento escrito).

\section{La práctica como investigación}

Sería impensable en esta sede revisar todas las metodologías que se incluyen bajo el paragua conceptual de investigación conducida por la práctica, por lo que me concentraré en el modelo que desde mi perspectiva resulta más atractivo y aplicable en nuestros contextos latinoamericanos denominado práctica como investigación. Esta metodología emergente se caracteriza por establecer que existe un tipo de conocimiento que surge desde los cuerpos y sus transformaciones lo que justificaría su aplicación a distintos campos disciplinarios donde "[l]a investigación tendría lugar en y a través de los cuerpos. Podría transmitir y proliferar entre los cuerpos, cuyas transformaciones podrían ser irreductibles, literalmente incomparables, eternamente singulares e irrevocable, no susceptibles de escritura" (JONES, 2009, p. 20, trad. mía). Esta investigación es eminentemente performativa y se diferencia radicalmente de la investigación cualitativa y cuantitativa. Tal como postula Brad Haseman en A Manifesto for Performative Research (2006) la característica distintiva de este tipo de investigación es que:

[...] se expresa en formas no numéricas y en forma simbólicas que van más allá de las palabras y los textos discursivos. Estas formas incluyen formas materiales de prácticas, de imágenes 
fijas o en movimiento, música o sonido, acción en vivo o códigos digitales" (HASEMAN, 2006, p. 6, trad. mía).

Si bien es cierto que en varias disciplinas sociales se ha producido una suerte de giro performativo que vincula investigación con práctica (como por ejemplo en la etnografía), lo característicos de las investigaciones performativas es que consideren la práctica como la principal actividad investigativa.

En primer lugar la investigación debe ser iniciada por la práctica, las preguntas, problemas y desafíos son identificados y formados por las necesidades de la práctica y los investigadores prácticos; en segundo lugar, la estrategia de investigación se desarrolla por medio de la práctica usando predominantemente metodologías y métodos específicos y conocidos para quienes hacen práctica (GRAY, 1996, p. 3, trad. mía).

La predominancia de la práctica no implica que todo lo que sucede en el curso de la investigación sea exclusivamente práctico, sino que más bien se favorece una triangulación entre tres tipos distintos de conocimiento: el conocimiento del investigador (que incluye conocimiento corporizado, experiencia fenomenológica, el saber hacer), la reflexión crítica (investigación-acción, conocimiento explícito) y conocimiento conceptual (entendidos como marcos teóricos) (NELSON EN JONES, 2009).

Desde mi perspectiva lo que define una práctica como investigación es que las preguntas o motivaciones iniciales solo puedan ser contestadas mediante la práctica. Esto implica que si bien los aspectos conceptuales y la reflexión crítica están presentes, lo crucial sigue siendo la práctica. Por ejemplo, entre los años 2008 y 2013 conduje una práctica como investigación que se interrogaba sobre los modos mediante los cuales el teatro puede recuperar, reconstruir y resemantizar la memoria. La investigación se titulaba "Teatro y memoria. Estrategias de (re) presentación y elaboración escénica de la memoria traumática infantil" y tenía como objetivo general explorar escénicamente las posibilidades de performativización de testimonios de personas que en Chile durante la dictadura eran niños ${ }^{2}$. Como es evidente, esta pregunta de investigación solo podía responderse mediante la experiencia de trabajo con actores. Junto a los actores probamos distintas modalidades de traducción escénica de los testimonios y era esta experiencia laboratorial lo que guiaba nuestro actuar, el diseño y el camino que, desde la práctica, debíamos seguir. En esta práctica como investigación fue crucial el trabajo 
interdisciplinario con Milena Grass (traductora y teórica del teatro) y Nancy Nichols (antropóloga), quienes aportaban una mirada crítico-reflexiva al proceso. Aun así lo que permanecía en el centro del proyecto era la propia práctica investigativa que de una forma u otra se conducía reflexivamente a sí misma.

\section{Aspectos Metodológicos}

La metodología de las investigaciones conducidas por la práctica tiene diferencias sustanciales con la metodología de la investigación clásica en las ciencias sociales. Tal como plantea Josette Féral (2009), la investigación clásica es de carácter analítico-descriptiva y ocupa herramientas de disciplinas sociales tales como la sociología, semiótica y la historia. Estas modalidades investigativas se han instalado hegemónicamente como la forma de realizar investigación considerando la práctica apenas como su objeto de estudio. En una investigación de esta naturaleza, existe una hipótesis, una fase de recogida de datos, una fase de análisis y la difusión se realiza mediante la publicación en revistas especializadas. Como veremos a continuación la práctica como investigación posee importantes diferencias respecto a esta aproximación hegemónica en nuestra academia.

Difieren también de las aproximaciones cualitativas y cuantitativas más convencionales. Según Brad Haseman (2006) muchas veces las prácticas como investigación no parten de una pregunta sino que se formulan a partir de un interés. Esto permite que el diseño metodológico exploratorio sea también más fluido y pueda emerger en el curso de la acción. No solo las preguntas iniciales, sino que también la hipótesis de investigación puede surgir a posteriori, siendo la propia práctica investigativa la que en un determinado momento ilumina su formulación. En una práctica como investigación la dosis de incertidumbre es mayor porque no todo puede formularse en palabras y, por tanto, no todo "aparece" en forma clara. Es por esto que el rol de la hipótesis es particular puesto que esta nunca puede ser del todo "probada" como sería por ejemplo en un experimento. Lo que queda probado a nivel de la experimentación es que se puede experimentar, que se pueden proponer nuevas ligazones, que es factible generar nuevas experiencias para dar cuenta de posibles respuestas que en general son únicas e idiosincráticas. 
La dosis de incertidumbre implica que a diferencia de aproximaciones más convencionales (cualitativas, cuantitativas, pero también por ejemplo de estudios teatrales) que necesariamente definen a priori un diseño de investigación que da cuenta de sus objetivos generales y específicos y de los procedimientos que se van a realizar, en la práctica como investigación este diseño es fuertemente "emergente" en el sentido que varia a medida que se desarrolla la proyecto. En este sentido la práctica como investigación se emparenta más con la investigación cualitativa, pero -y aquí insisto sobre la dificultad de traducibilidad discursiva de las prácticas- esta emergencia se torna más radical, puesto que implica soportes plurimatéricos y polisensoriales.

La práctica como investigación requiere entonces un delicado equilibrio que no es para nada fácil. Se trata por un lado de tener siempre en mente las preguntas de la investigación (que funcionan como timón del trabajo), pero a la vez tener la suficiente flexibilidad para acoger aquello que surge desde la práctica. El equilibrio resulta complicado y exige lo que defino como una mirada binocular que logre mirar una misma cosa desde dos perspectivas distintas en forma simultánea. La práctica como investigación necesita investigadores entrenados para lograr esta fusión binocular que pueda aunar las perspectivas corpóreas y discursivas construyendo un objeto único.

En esta visión binocular resulta imprescindible la documentación del proceso. Al igual que en la denominada investigación-creación (FÉRAL, 2009; SÁNCHEZ, 2009) es crucial que los investigadores-prácticos lleven un registro del proceso. Este registro puede ser escrito, pero también incluir videos, imágenes, dibujos y otros tipos de materialidades. Estos materiales sirven después para reconstituir analíticamente la genética del proceso de investigación. Tal como plantean Ledger, A., S.K. Ellis y F. Wright (2011) los registros pueden ser de diversa índole para abarcar distintas estrategias de archivización de las prácticas. Estos materiales resultan después fundamentales para la comprensión de lo que ha sucedido en el proceso investigativo. El registro en estos casos no se limita a describir el proceso sino que también funciona como dispositivo que transmite conocimiento. Tal como plantea Barrett (2010) la exégesis puede entenderse como un meme, es decir como un dispositivo que es a la vez una idea y el vehículo del conocimiento. La exégesis no se limita a describir sino que aspira a dilucidar los procedimientos para generar otras formas de conocimiento en el contexto académico potenciando la metodología que describe y encarna más allá de ese trabajo en particular. 
"La exégesis puede ser vista tanto como una réplica o una revisión del trabajo artístico completo como también un discurso reflexivo sobre momentos significativos de revelamiento" (BARRETT, 2010, p. 160, trad. mía).

Otra importante característica distintiva es que los resultados de prácticas como investigación deben saber reflejar esta visión binocular e incluir en sus resultados dispositivos prácticos sean estos una obra, un taller o una experiencia intersubjetiva programada. No me parece, como hacen algunos investigadores prácticos, que sea conveniente descartar como resultado la escritura a priori, muchas veces hay aspectos del resultado práctico que son susceptibles de verbalización y que puedan por ende ser formulados en términos de un paper. Es imprescindible, sin embargo, que cuando se incluya un documento escrito (sea este un paper, una exégesis o una memoria de obra) se explicite que la operación de transducción intersemiótica es penalizante para la riqueza multisensorial y experiencial propia de la práctica. Es decir que se explicite y reconozcan los límites del discurso para dar cuenta de la compleja multimodalidad de las prácticas.

Una posibilidad interesante, son los denominados ensayos performáticos que "invitan a una reconceptualización experiencial" (NELSON, 2009, p. 118). Este formato, escasamente explorado en la academia de nuestro país, entreteje un argumento con una forma expresiva generalmente no verbal que de cuenta del postulado. Se parte de un argumento (afirmación o postulado) que debe ser probado tanto discursivamente como en la práctica. Por ejemplo, en el curso "Políticas y estéticas del cuerpo en escena" del Magíster y Doctorado en Artes UC que dicté en 2013 les pedí a los estudiantes que hicieran un ensayo performativo como examen final. Uno de los argumentos, del alumno José Miguel Candela formuló el siguiente argumento:

El uso de la tecnología digital en su relación con el cuerpo comprueba una doble dimensionalidad de características dialécticas. Si bien encarna la evolución del cuerpo hacia un pos-humano tecnológico y a una posible emancipación, provocando así una modificación fenomenológica del cuerpo, también despliega, gracias a la misma tecnología, un tipo de vigilancia panóptica (de los observados, de los que vigilan), que la evolución tecnológica perfecciona y que el poder económico ejerce, y que en líneas generales se desarrolla para la obtención de más y mejor información, y finalmente, de mayor productividad (CANDELA, 2013, trabajo entregado para el curso). 
Junto con un interesante trabajo escrito, José Miguel realizó una instalación. En una sala estaba una bailarina cuyos movimientos eran traducidos por un software como sonidos musicales. Los espectadores entramos a la sala y presenciamos la instalación durante algunos minutos. Poco a poco nos fuimos percatando que había cámaras en la sala que no estaban enfocadas en la bailarina sino en nosotros como espectadores. Descubrimos que en la sala contigua un circuito cerrado "nos vigilaba" grabando todo lo que hacíamos en la instalación. Esta sala contigua tenía también un computador donde aparecía información extraída de internet sobre los espectadores. Esta experiencia performativa nos permitió como participantes vivenciar el argumento de José Miguel en nuestros cuerpos, lo que después se tradujo en una conversación muy interesante que sin duda habría sido distinta sin la experiencia práctica. Los resultados de una práctica como investigación deben rescatar la visión binocular antes mencionada y descubrir la forma de transmitirlo al espectador/lector/destinatario de la investigación. Cuando esta operación es exitosa, la valencia política de la práctica como investigación se irradia y circula más allá del territorio académico.

La práctica como investigación, en fin, adhiere a una ética y política de investigación. En este sentido el hecho de ser emergente o dinámica no implica que cualquier tipo de experiencia pueda considerarse práctica como investigación. La práctica como investigación no es lo mismo, por ejemplo, que la indagación propia del quehacer artístico. Es bien sabido que antes de empezar a montar una obra de teatro o a diseñar una instalación el artista se prepara: lee, piensa, diseña, discute. Estos procesos indagatorios no son equiparables a la lógica de la investigación en la academia a la que la práctica como investigación desea de alguna forma, aunque sea parcialmente, adscribir. La diferencia radica en que la práctica como investigación exige tener claras las preguntas y diseñar (aunque esto varíe en el camino) una cierta metodología. Las preguntas, al igual que en la investigación científica deben ser específicas para lograr orientar el proceso. Una buena pregunta delimita el ámbito de acción performativa y encuadra la experimentación. Además, en una práctica como investigación lo más relevante es el proceso de indagación y la experimentación: el fin último es explorar, no crear. En eso difiere de la indagación artística que busca crear una obra, un producto artístico. En la práctica como investigación el propósito es autorreflexivo y busca generar nuevo conocimiento (encarnado, práctico), no (necesariamente) crear una obra de valor estético (aunque esto también puede darse). Otro aspecto fundamental que no siempre se verifica en el trabajo de los artistas es el 
registro del proceso. Tal como mencioné anteriormente, el registro es fundamental a la hora de realizar una práctica como investigación. Este registro, a diferencia del registro que puede llevar un artista (cuaderno de notas, bitácora, dibujos, bosquejos), responde a parámetros de legibilidad para que después puedan ser analizados, re-articulados, re-presentados para reconstruir la genética del proceso investigativo.

\section{Implicancias políticas y epistemológicas}

La práctica como investigación no es solo una propuesta metodológica, se trata, en el fondo, de un proyecto político.

Sus fundamentos epistemológicos ponen en evidencia un deseo profundo de modificar la visión dominante de la investigación más común en el Reino Unidos y los Estados Unidos. Va acompañada de un objetivo político, radical con la voluntad de intervenir la sociedad [...] Sus objetivo, métodos y finalidad modifican nuestra mirada sobre la investigación y su papel" (FÉRAL, 2009, p. 325).

En este sentido, y tal como plantea Kershaw (2009) en las últimas décadas se ha hecho cada vez más claro que la práctica como investigación puede desencadenar desafíos fundamentales y radicales a los paradigmas sobre el conocimiento. Esto es algo que se ha verificado en las universidades que han implementado estas metodologías conducidas por la práctica que han podido contribuir significativamente a la academia en general (JONES, 2009). Esta perspectiva político-metodológica permite en buenas cuentas avanzar un paso más allá en la crítica a la colonización de la mirada objetivante típicamente científica que ha primado en nuestras universidades.

La práctica como investigación es también un efectivo antídoto a lo que Michel Foucault llamó el disciplinamiento de los saberes que implica la normalización, distribución y jerarquización el conocimiento. Esta aproximación no solo discute la hegemonía del imperialismo textualizante sino que también, por trabajar en el campo de las prácticas, relativiza los dominios disciplinarios promoviendo una antidisciplina que pone en juego las distinciones, calificaciones y normativas del disciplinamiento epistémico eurocéntrico imperante. Tal como explica Foucault en Vigilar y Castigar (1975) la disciplina, en cuanto mecanismo de regulación de la conducta implica entre otras consecuencias la selección, normalización, jerarquización y centralización 
de los contenidos del saber lo que redunda en el control de la producción de discurso. Para hablar/producir/compartir saber, se debe seguir un largo camino de validación que sigue un tiempo disciplinario que busca a fin de cuentas la distribución del conocimiento. Esta estructura disciplinar de los saberes, tan propia del siglo XX, se ve amenazada por las metodologías de práctica como investigación que cercenan una de las fronteras más férreas en la academia la división entre conocimiento intelectual y saber práctico. Pero además la práctica como investigación desdibuja las fronteras disciplinarias, puesto que requiere que las disciplinas colaboren en una configuración posdisciplinar.

Para ejemplificar el borramiento de la distinción entre fronteras disciplinarias, volveré sobre la práctica como investigación antes mencionada que pretendía explorar estrategias de escenificación de los testimonios de personas que habían sido niños en la dictadura en Chile. Para tal efecto, la antropóloga y experta en historia oral Nancy Nichols elaboró una entrevista semiestructurada. El trabajo práctico con actores fue develando que muchos recuerdos de niños se asientan en experiencias sensoriales más que en episodios o eventos. A partir de este "descubrimiento" Nancy decidió incluir preguntas en la entrevista que apelaran a estos recuerdos sensoriales como por ejemplo: ¿qué olor recuerdas?; si el día del golpe fuera un color, ¿qué color sería?. Es así como en la estructuración de la entrevista colaboraron distintas disciplinas difuminando las fronteras del saber. De esta forma la práctica influyó directamente en la construcción del instrumento afectando y cambiando una técnica tradicional de construcción de entrevistas.

\section{Aplicabilidad del modelo en Chile y América Latina}

Las metodologías guiadas por la práctica se han validado en el campo de los estudios de postgrado anglosajones. Para concluir me gustaría reflexionar sobre la aplicabilidad del modelo en Chile pensando en las artes y cómo desde aquí se podría operar una irradiación hacia otras disciplinas. En primer término conviene revisar el contexto geopolítico de Chile en cuanto país latinoamericano e identificar las diferencias respecto a los contextos europeos o norteamericanos. Es cierto que la relación con los cuerpos en América Latina es distinta respecto a otros territorios. Aquí, la racionalidad y el cientificismo eurocéntrico se encontraron con otros modos de experienciar el cuerpo que, de una u otra forma, entraron en diálogo con la estricta división 
cartesiana. Esta herencia precolombina es tal vez una de las razones por la que en varios países de América Latina las escuelas de formación de artistas se instalaron en las universidades a diferencia de lo que sucede por ejemplo en Europa donde los artistas tradicionalmente se han formado en academias extrauniversitarias. No creo que la forma como en América Latina hemos históricamente articulado la teoría y la práctica pueda ser del todo comparable con lo que ha sucedido tradicionalmente en Europa o Norteamérica. El contexto latinoamericano ha permitido otro tipo de relación con los saberes del cuerpo. La academia universitaria, sin embargo, no ha sabido preservar ese patrimonio intangible adhiriendo con el paso del tiempo casi acríticamente a los parámetros impuestos desde otros continentes. Vemos como hoy en las universidades la consolidación de la implacable tiranía de los papers, los ISI, las ponencias en congresos e, incluso, el uso cada vez más frecuente del inglés como idioma de postulación a los fondos nacionales de investigación (como por ejemplo el Fondecyt). Desde mi perspectiva la globalización en ese sentido ha sido implacable. Sus víctimas, el cuerpo y sus saberes, han quedado relegados a ciudadanos de segunda clase.

Las metodologías conducidas por la práctica desarrolladas en el Reino Unido, Sudáfrica, Australia y Estados Unidos adhieren a un proyecto político descolonizador que pretende justamente devolver al cuerpo su posibilidad de acción. Este proyecto político aunque criado fuera de nuestros territorios se emparenta con la necesidad en nuestros países de repensar la forma cómo educamos a nuestros niños, cómo se genera conocimiento, y con qué criterios se valida el conocimiento en nuestra sociedad. La práctica como investigación permite valorar y validar los saberes del cuerpo, su accionar, su creatividad, su sensorialidad y psicomotricidad, no como saberes ornamentales sino como saberse fundantes de nuestra subjetividad. Mi interés en esta apuesta metodológica responde entonces a ese proyecto descolonizador del racionalismo acérrimo, del cientificismo, de la objetivación de los cuerpos.

Se requiere, sin embargo, que quienes estamos en la academia nos comprometamos con ese proyecto político activamente para influir en las políticas públicas que regulan la universidad y la investigación en nuestro país. El Fondecyt (Fondo Nacional de Investigación en Chile), por ejemplo, veta en sus bases la posibilidad de trabajo con artistas lo que necesariamente impide el desarrollo de prácticas como investigación en el ámbito de las artes. El formulario resulta igualmente restrictivo en lo que compete a otras disciplinas que deben responder a un diseño clásico que no admite otras metodologías. Los resultados exigidos, en cualquier 
caso se reducen a publicaciones ojalá en revistas ISI en inglés, y excluyen cualquier otro tipo de resultado de índole práctica que queda relegado en el informe final a "logro." Es necesario que los académicos involucrados en el trabajo de investigación práctica avancemos hacia la promoción de políticas públicas que poco a poco equiparen los derechos/deberes con los de la investigación clásica.

\section{Una primera experiencia en Chile: el Doctorado en Artes de la Universidad Ca- tólica}

Recientemente se ha inaugurado el Doctorado en Artes UC en el que participo como docente. Después de varios años de trabajo de la Comisión de Diseño del Doctorado en Artes UC se formuló un programa que admite entre sus posibles salidas la realización de una práctica como investigación. Esto quiere decir que los candidatos pueden elegir si realizar una tesis convencional (en historia de las artes, semiótica, estudios teatrales, etnomusicología, etc.) o bien aventurarse en la ardua tarea de explorar metodologías conducidas por la práctica. En estos casos los requisitos para la obtención del grado contemplan no solo la redacción de un documento escrito (tesis), sino también la realización de una obra o dispositivo práctico que dé cuenta del trabajo desarrollado. Se trata de un programa innovador que intenta avanzar en la descolonización de la teoría hacia metodologías conducidas por la práctica. En este programa el Comité de Postgrado definió las tres opciones que justifican una modalidad de tesis doctoral que incluye obra:

1. La hipótesis de investigación solo puede ser validada por medio de la práctica artística.

2. Cuando la obra constituye una respuesta, aunque sea parcial, a la pregunta de investigación.

3. Cuando la determinación de la hipótesis surge a partir del proceso de creación.

Destaco este incipiente modelo que, sin duda, deberá ajustarse en el proceso de implementación por su coraje para proponer un giro epistémico que no se contenta con teorizar el cuerpo sino que busca ampliar el horizonte epistémico a través del cuerpo. Se trata de un programa piloto en nuestro país (y también en América Latina) que espero permita, en la práctica, probar la aplicabilidad de estas metodologías en Chile. 


\section{Lo que el cuerpo sabe}

En fin, para concluir es necesario reivindicar que aquello que el cuerpo sabe no puede traducirse al discurso, no del todo por lo menos. Existe un residuo de la experiencia que habita en nuestro cuerpo individual y también en nuestros cuerpos sociales que es irreductible a la palabra. Los saberes del cuerpo son otros tipos de conocimientos que pueden ampliar, airear, expandir los horizontes epistémicos a los que la academia eurocéntrica nos ha acostumbrado.

\section{Notas}

1 La reflexión sobre las prácticas no es del todo nueva, existen importantes aportes desde la filosofía (Wittgeinstein 1953; Taylor 1985), la sociología (Bourdieu 1990; Giddens 1979; Latour 2005), la teoría crítica (Foucault 1975), así como también de la semiótica que hace ya varias décadas ha puesto en relieve la importancia de la emergencia de significados desde las prácticas. (Fabbri 2005; Fontanille 2006). 2 Para una descripción exahustiva de este trabajo véase Contreras, Grass y Nichols 2009.

\section{Referencias}

ALLEGUE, L.; KERSHAW, B.; JONES, S.; PICCINI, A. (eds.). Practice-as-Research in Performance and Screen. Basingstoke: Palgrave, 2009. BARONE, T.; EISNER, E. Arts-based educational research. In: JAEGER, R. M. (ed.) Complementary Methods for Research in Education, 2nd Edition. Washington: American Educational Research Association, 1997, p. 72-116.

BOURDIEU, P. The Logic of Practice. Cambridge: Polity Press, 1990.

COLE, A.; NEILSEN, L.; KNOWLES, G.; LUCIANI, T. (eds.). Provoked by Art: Theorizing Arts-Informed Research. Halifax, NS: Backalong Books, 2004

CONTRERAS, M.J.; GRASS, M.; NICHOLS, N. (2008). Pajarito Nuevo la Lleva: estratégias de representação e elaboração cênica de memória traumática infantil. Revista Aletria: Revista de Estudos de Literatura, Artes e Cultura de Expressão Hispânica. Minas Gerais: Universidade Federal de Minas Gerais, v. 17, p. 154-172, 2009.

EISNER, E.W. Art and Knowledge. In: KNOLES, G.; COLES, Al. Handbook of the Arts in Qualitative Research: Perspectives, Methdologies, Examples and Issues. California: Sage, 2007, p. 3-12

FABBRI, P. La svolta semiótica. Bari-Roma: Laterza, 1998.

FÉRAL, J. Investigación y creación. Estudis Escénics. Quaderns del'Institut del Teatre, n 35, p. 327-335, 2009.

FONTANILLE, J. Pratiche Semiotiche. Semiotiche. Testo, pratiche, immanenza, n. 4, p. 161-185, 2006.

FOUCAULT, M. Vigilar y castigar. Trad. de Aurelio Garzón. Madrid: Siglo Veintiuno Editores, 1975. 
FRAYLING, C. Practice-Based Doctorates in the Creative and Performing Arts and Design. Lichfield: UK Council for Graduate Education, 1997

GIDDENS, A. Central Problems in Social Theory. California: University of California Press, 1979.

GRAY, C. Inquiry through Practice: Developing Appropriate Research Strategies. 1996. Recuperado el 20 julio 2013. http://carolegray. net/Papers\%20PDFs/ngnm.pdf

HASEMAN, B. C. A Manifesto for Performative Research. Media International Australia Incorporating Culture and Policy: Quarterly Journal of Media Research and Resources, v. 118, p. 98-106, 2006.

JONES, S. The Courage of Complementarity: Practice-as-Research as a Paradigm Shift in Performance Studies. In: ALLEGUE, L.; KERSHAW, B.; JONES, S.; PICCINI, A. (eds.) Practice-as-Research in Performance and Screen. Basingstoke: Palgrave, 2009, p. 18-32.

KERSHAW, B. Introduction. In: ALLEGUE, L.; KERSHAW, B.; JONES, S.; PICCINI, A. (eds.). Practice-as-Research in Performance and Screen. Basingstoke: Palgrave, 2009, p. 1-16.

LATOUR, B. Reassembling the Social: An Introduction to Actor-Network-Theory. Oxford: Oxford University Press, 2005.

LE BRETON, D. Antropología del cuerpo y modernidad. Trad. Paula Mahler. Buenos Aires: Nueva Visión, 2002.

LEDGER, A.; ELLIS, S.K.; WRIGHT F. The Question of Documentation: Creative Strategies in Performance Research. En: KERSHAW, B.; NICHOLSON, Helen (eds.). Research Methods in Theatre and Performance. Gran Bretaña: Edinburgh University Press, 2011, p. $162-185$.

NELSON, R. Modes of Practice-as-Research Knowledge and their Place in the Academy. En: ALLEGUE, L.; KERSHAW, B.; JONES, S.; PICCINI, A. (eds.). Practice-as-Research in Performance and Screen. Basingstoke: Palgrave, 2009, p. 112-131.

SÁNCHEZ, J. A. Investigación y experiencia. Metodología de la investigación creativa en artes escénicas. Estudis Escènics. Quaderns del'Institut del Teatre, n. 35, p. 327-335, 2009.

SCHATZKI, K.; KNORR, C.; VON SAVIGNY, T. (eds.). The Practice Turn in Contemporary Theory. London: Routledge, 2001.

SULLIVAN. Research Acts in Art Practice. Studies in Art Education, n 48, p. 19-35, 2006.

TAYLOR, C. Human Agency and Language. New York: Cambridge University Press, 1985.

VERA, A. Arte y conocimiento: algunas reflexiones desde la perspectiva del postgrado Cátedra de Artes, n 8, Santiago, Chile, p. 9-28, 2010. 